\title{
Navigation in Degree of Interest Trees
}

\author{
Raluca Budiu \\ budiu@parc.com \\ Palo Alto Research Center \\ 3333 Coyote Hill Rd. \\ Palo Alto, CA 94304
}

\author{
Peter Pirolli \\ pirolli@parc.com \\ Palo Alto Research Center \\ 3333 Coyote Hill Rd. \\ Palo Alto, CA 94304
}

\author{
Michael Fleetwood \\ fleet@rice.edu \\ Rice University \\ P.O. Box 1892 \\ Houston, TX 77005
}

\begin{abstract}
We present an experiment that compares how people perform search tasks in a degree-of-interest browser and in a Windows-Explorer-like browser. Our results show that, whereas users do attend to more information in the DOI browser, they do not complete the task faster than in an Explorer-like browser. However, in both types of browser, users are faster to complete high information scent search tasks than low information scent tasks. We present an ACT$\mathrm{R}$ computational model of the search task in the DOI browser. The model describes how a visual search strategy may combine with semantic aspects of processing, as captured by information scent. We also describe a way of automatically estimating information scent in an ontological hierarchy by querying a large corpus (in our case, Google's corpus).
\end{abstract}

\section{Categories and Subject Descriptors}

H.52 [User Interfaces]: Theory and methods; D.2.8 [Software Engineering]: Metrics-complexity measures, performance measures

\section{General Terms}

Measurement, Human Factors, Theory

\section{Keywords}

User studies, user models, ACT-R, DOI trees, information scent, information visualization

\section{INTRODUCTION}

Visualizing large hierarchical information structures is a pervasive task in the real world. Whether the structures are organization charts, web pages, chronological information, or even file systems, there is a need of being able to see as much and as global a view as possible on a limited size screen. With that aspiration in mind, one of the most successful and inventive techniques was focus + context [3]. Focus + context is a name that covers several visualization methods aiming to increase the amount of information displayed to a user. Such visualizations generally achieve this by presenting in greater detail the information around the user's (assumed) point of focus and by offering a more schematic view of the other parts of the information structure. Fisheye views [3], hyperbolic trees [7], and degree-of-interest trees [4] are all focus + context techniques.

However, in spite of the intuitive efficacy and of the enthusiasm generated in the user-interface community, it is not clear that focus + context techniques present a real advantage to the everyday user. Performance in focus + context visualization appears to be largely a function of the relation between the semantics of the navigation cues and the user's tasks. This relation is called information scent and is described in greater detail below. For instance, [13] looked at users' performance on search tasks when they used a hyperbolic tree browser and a more common, Windows Explorer-based browser. They concluded that, although in some cases (e.g., expert users or low information scent tasks) the hyperbolic tree may produce faster results, the interaction between the semantic qualities of the labels and the visual layout is quite complex and a clear advantage cannot really be predicted for the hyperbolic tree. Indeed, one of the first questions that confronts anybody trying to understand how users perform the search tasks in these focus + context browsers is how do users choose what to focus their attention on? Are the visual cues (e.g., node density, color, arrangement on page) what determine focus, or are the semantic features of the display (is this label related to what I am looking for) more likely to dominate? Are visual cues more likely to matter when the information scent (that is, the semantic quality of the labels) is poor than when it is strong?

The purpose of this paper is to understand how the combination between visual and semantic information in the focus + context browser affect users' performance in a search task. To achieve this goal, we first collected experimental data about how users perform simple search tasks in a type of focus + context browser called degree-of-interest (DOI) tree. We looked at two kind of search tasks: high-scent tasks, in which the target item was on a highly predictable, commonsense path, and low-scent tasks, in which the target item was hidden under less plausible labels. We collected the times that people needed to complete the search tasks for two different browsers, as well as finer grain eye-movement data such as number of nodes looked at, number of revisitations per node, or direction of eye gaze. Then, based on these data, we built a detailed cognitive model of navigation in 
DOI trees. The cognitive model took into account complex visual search phenomena, as well as semantic aspects of the information processing. One of the advantages of cognitive modeling is that it provides a very well defined theory of the process that users go through; based on that theory, the interface designers can make low-cost predictions about new designs that conform to the assumptions of the model and they can also evaluate the quality of their design based on those features that the model deems most determining of human performance.

One of the problems in evaluating interfaces or in devising computational models of browsing is an automatic measure of information scent: how do we know which nodes look highly relevant to people? Often designers' intuitions do not match the other people's intuitions. This paper describes a way of automatically determining scent in an ontological hierarchy. Our method essential involves extracting category relationships from a large corpus (in our case the corpus that Google makes accessible through their API ).

Next we briefly review two concepts that are central to this study: degree-of-interest trees and information scent.

\subsection{Degree of Interest Trees}

Degree of Interest (DOI) trees were introduced first by [4]; they are an instance of focus + context visualization and use degree-of-interest calculations to decide what gets displayed on the screen. Figure 1a shows a degree of interest tree that represents an ontological hierarchy of concepts. The hierarchy was first used in the Great CHI'97 Browse-off [11]. In Figure 1 the node in focus is Artificial, the node that was last clicked by the user. Note that only the nodes close (in terms of tree distance) to the node in focus or to its ancestors are displayed. For instance, the children of the node Science are not displayed in this image because they are at a distance higher than 2 from the path from root to the node in focus. Also note that, whereas various parts of the tree are shown in different detail, the overall structure of the tree is also visible.

Compare this browser with a traditional Windows-Explorerlike type of file browser (henceforth called Explorer). Figure $1 \mathrm{~b}$ shows the image that the user sees after clicking on the node Artificial in an Explorer browser. Fewer nodes are displayed in this image and the user cannot really get any sense about the kind of information hidden under the other labels except from the ones he has already clicked. The tree structure looks harder to grasp in this display too.

The assumptions underneath the DOI tree visualization are (1) that the users will navigate more easily through the information structure because its skeleton is clearly exposed; and (2) that they will learn faster about the information in the tree, because more (possibly unnecessary) information is available to them.

\subsection{Information Scent}

Throughout the paper we refer to the following search task: find the node with the label $A$ in a hierarchical ontology, as displayed by some browser. We sometimes abbreviate this task as find $A$. $A$ is called the target node. In solving this task, the user has to click on a sequence of nodes to find the descendants of those nodes that are not already displayed on the screen.

To understand how the semantic quality of the labels affects the navigation, we looked at two kinds of tasks that differed in how predictable the path to the target was: tasks with high information scent and tasks with low information scent. Information scent [12] is the "perception of the value, cost, or access path of information sources, obtained from proximal cues such as bibliographic citation, WWW links, or icons represented the sources." In other words, in a search tasks, the information scent of a label is an informal estimate of the likelihood that the label hides the target node. The information scent captures the semantic aspect of the decision making in search. What does it mean for a search task to be high or low scent? A task is high scent if all the labels on the path to the solution have high scent; it is low scent if at least some of these labels are low scent. For instance, for the search task find a banana in an ontology like the one in Figure 1, the path to the node banana is Categories $\rightarrow$ Things $\rightarrow$ Natural $\rightarrow$ Vegetable $\rightarrow$ Fruits $\rightarrow$ Tropical $\rightarrow$ Banana, so the task is high scent because all nodes on the path make sense. However, our ontology is not perfect: an item such as Library of Congress is under Categories $\rightarrow$ People $\rightarrow$ Specific People $\rightarrow$ Organizations $\rightarrow$ Governmental $\rightarrow$ United States $\rightarrow$ Legislative Branch $\rightarrow$ Library of Congress; this task is low scent because it is less intuitive that Library of Congress should be under the labels People or Specific People. Because intuition is hard to capture, in our experiments we actually use human ratings to decide whether a task is low or high scent.

\subsection{Overview}

In the remainder of the paper we describe an experiment in which we collected data about people browsing through a DOI browser and an Explorer browser; next we discuss a way to calculate automatically information scent, and finally, we present our cognitive model for DOI tree navigation.

\section{EXPERIMENT}

As discussed in the introduction, the DOI tree is a clever visualization that seems to promise substantial performance gains for its users. In this experiment we wanted to gather data to support or invalidate this hypothesis. Specifically, we looked at how participants performed low- and high-scent search tasks using two browsers: a DOI browser (see Figure 1a) and a traditional, Explorer browser (see Figure 1b). We collected a multitude of measures for these tasks: reaction times, mouse movements and clicks, eye movements, but we do not discuss all of them in this article.

\subsection{Method}

Participants. Eleven participants were recruited from Stanford University and from PARC; the participants from Stanford were paid $\$ 40$. Two additional recruits were eliminated due to eye tracking problems.

Apparatus. We used the two browsers introduced before: the DOI browser and the Explorer browser. An ISCAN RK426PC eye-tracker was used to record eye movements.

Materials. Thirty two search tasks were used. All these tasks involved finding one or more nodes in the ontologi- 


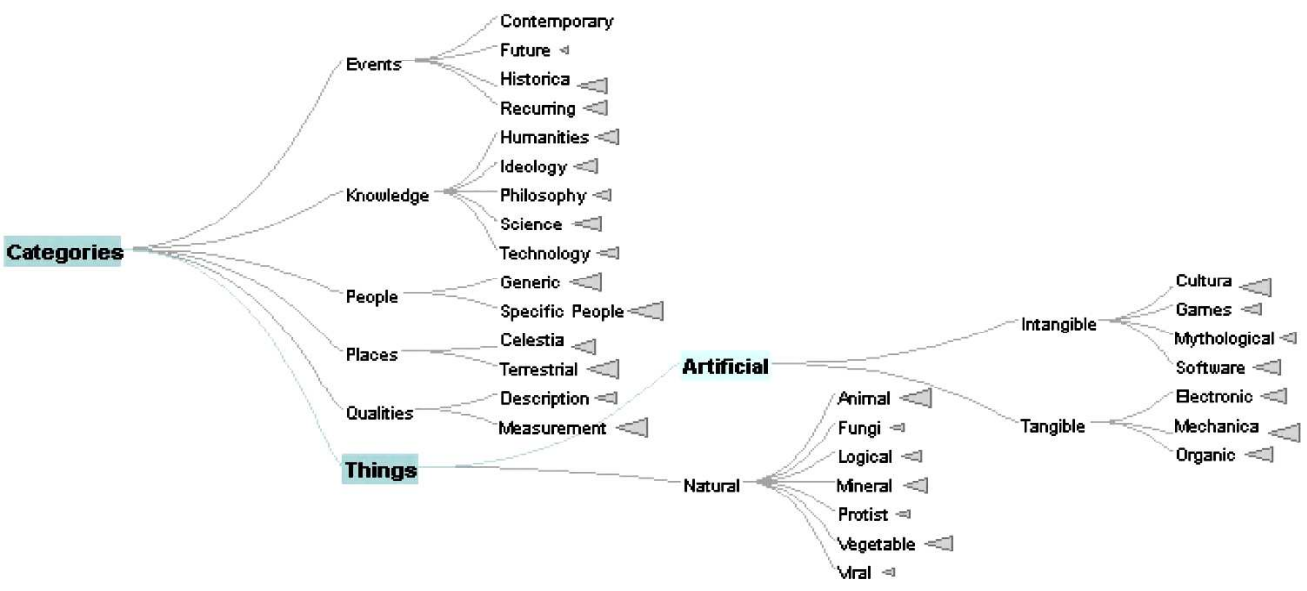

(a)

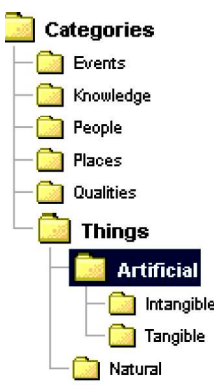

(b)

Figure 1: Two different visualizations for the same ontological hierarchy: (a) DOI tree (b) Explorer.

cal hierarchy used in [13]. In this paper we only discuss the 16 tasks that involved finding a single node in the hierarchy. Out of the 16 tasks, 8 were low scent and 8 were high scent. Examples of low scent tasks include find the Library of Congress (discussed in Section 1.2), find the play Romeo and Juliet (the path to that node was Categories $\rightarrow$ Things $\rightarrow$ Artificial $\rightarrow$ Intangible $\rightarrow$ Cultural $\rightarrow$ Literature $\rightarrow$ Examples $\rightarrow$ Plays $\rightarrow$ Shakespeare $\rightarrow$ Romeo and Juliet). Beside the banana example discussed in Section 1.2, other instances of high-scent tasks were: find the Ebola virus (under Categories $\rightarrow$ Things $\rightarrow$ Natural $\rightarrow$ Viral $\rightarrow$ Ebola $)$ and find the lobster (under Categories $\rightarrow$ Things $\rightarrow$ Natural $\rightarrow$ Animals $\rightarrow$ Invertebrates $\rightarrow$ Arthropods $\rightarrow$ Crustaceans $\rightarrow$ Lobster). All these tasks were used before in Experiment 1 from [13]. The decision whether a task was high or low scent was based on normative data collected in [13]; in that study participants were asked to report their familiarity with each of the tasks and also to locate where the targets are more likely to be found in the first four levels of the Great CHI Browse-off ontology. The information scent score was defined as the proportion of participants who correctly identified the location of the target item from looking at the four upper tree levels. All the low scent tasks in our study had an information score lower than 0.17 ; all the high-scent tasks had an information score higher than 0.32 . The number of steps to the solution (i.e., solution path length) did not differ significantly for the two types of tasks (7.87 steps for high scent and 8.25 steps for low scent; $t(14)=-0.35, p>0.7)$.

Procedure. The participants proceeded through (a) a familiarization phase, (b) a practice phase, and (c) a test phase. During the familiarization phase, the experimenter walked the participant through two introductory practice tasks demonstrating the basic functions of each browser. During the practice phase, each participant completed two practice tasks with each browser. The practice tasks were conducted in the same manner as the experimental tasks, but were not timed. After the practice tasks, the participants' eyes were tracked. A brief session was devoted to calibrating the tracking system along a 9-point grid. During the test session, each participant completed the two sets
Table 1: Average response times (s) for the search tasks.

\begin{tabular}{|l|l|l|}
\hline Browser & High Scent & Low Scent \\
\hline DOI & 24.54 & 69.01 \\
\hline Explorer & 28.42 & 59.83 \\
\hline
\end{tabular}

of 32 tasks. For each participant, one test list was presented with one browser, and then the second test list of 16 items with the other browser. List order and browser order were counterbalanced across participants. The presentation order of test items within each list was randomized for each participant.

\subsection{Results and Discussion}

Our design was a repeated measure design. As suggested by Lorch and Myers [10], in order to make sure that all the error terms are taken into account, the correct statistical method to analyze this kind of design is to run separate regressions on the data from each subject that include all the variables of interest. Then, for each variable, one should use a t-test to check if the regression coefficients from all subjects are significantly different from 0 .

Response Times. Response times for trials in which the participant did not complete the task or were greater than 300 s were replaced with the participant's mean response time. In what follows we only report analyses for the 16 tasks that involved a single retrieval.

The average response times are shown in Table 1 . The regressions indicated that the only significant variable that affected the response times was the scent $(t(10)=3.75, p<$ 0.05): participants completed the high scent tasks faster than the low scent tasks. The browser had no effect on how fast the participants performed the tasks $(t(10)=-1.23, p>$ 0.2 ). Moreover, unlike in studies of the hyperbolic browser, where a slight facilitation was found for low scent tasks [13], there was no interaction between the scent and the browser type; namely, participants did not behave differently for dif- 
ferent types of tasks in different browsers. The lack of a browser effect contradicted the common sense expectation that the DOI tree may actually facilitate search due to more nodes being exposed to the participants.

In what follows, we explore some other measures of participants' performance in an attempt to understand why no performance difference was obtained for the two browsers.

Number of nodes visited. When we looked at the number of nodes visited (i.e., number of distinct nodes either looked at or clicked on) by each participant in each tasks, we found that there were far fewer nodes visited in the Explorer browser (on average, 53 nodes per task) than in the DOI tree browser ( 80 nodes). This effect was significant $(t(10)=-4.47, p<0.005)$. Also, the number of nodes visited in the high scent task was lower than for the low scent tasks ( 47 versus 85 nodes: $t(10)=4.21 ; p<0.005$ ), confirming our intuition that the low scent tasks were harder and that the participants had to wander around quite a while until they found the correct path. The interaction between browser and scent was not significant.

Finding that people visit more nodes in the DOI tree is not surprising: by definition, the DOI tree attempts to expose the users to as much as possible relevant information. However, the fact that they are not faster with DOI browser suggests that this extra amount of information possibly means time needlessly spent.

Average Revisitation. We also looked at how many times a participant revisited the same node on average. People tended to revisit more nodes in the Explorer than in the DOI browser $(t(10)=4.34 ; p<0.005)$ : on average they revisit a node about 6.6 times in the DOI tree, as indicated by the mouse clicks and eye movements. The same number is 8.15 for the Explorer. Since each revisit costs the participants time and based on the number of nodes visited, one can hypothesize that the time spent in the Explorer for revisitation (needed to backtrack when a wrong path has been taken) is actually used in the DOI browser for visiting new nodes. So in fact people do get some extra knowledge of the information structure with the DOI tree; however, getting that extra knowledge acts as a distraction at the level of task completion.

Distance to Solution Path. Since participants tended to wander more (i.e., visit more nodes) in the DOI browser, we wanted to see how far apart from the solution they actually got. We looked at the average distance from the nodes visited to the actual solution path ${ }^{1}$. Not surprisingly, participants went further away from the solution path in the DOI browser (on average, 4.57 nodes away) compared to the Explorer browser (only 3.96 nodes away). This difference was significant $(t(10)=-6.59 ; p<0.0001)$. Moreover, in the high scent tasks participants wandered less from the solution path (3.62 nodes on average) compared with the low scent tasks (4.83 nodes). The scent effect was also significant $(t(10)=4.2 ; p<0.005)$.

\footnotetext{
${ }^{1}$ By distance from a node to a path in the tree we mean the length of the minimum path from that node to a node on the path to the solution.
}

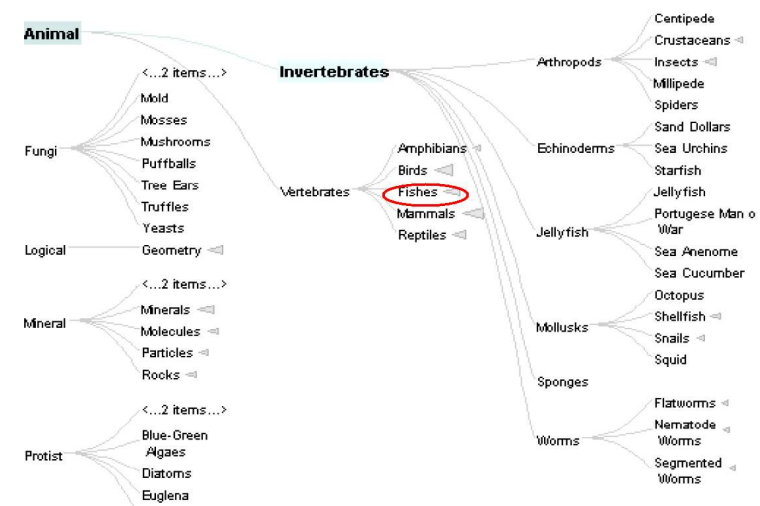

Figure 2: The node Fishes acts as a distractor in the task Find a lobster.

By examining these data and also the individual participants' data we got more insights into the advantages and disadvantages of the DOI tree. First, the DOI tree seemed to be more forgiving if participants made a mistake. Namely, even if the participants clicked on a wrong node, if the wrong node was close to the correct one, clicking on that node would also expand some of the children of the correct node and would offer the opportunity to recover from that mistake. For instance, in Figure 1a, the participant looking for the Ebola virus wrongly clicked on the node Artificial. However, that click also expanded the children of the correct node (Natural), thus giving the participant the occasion to correct themselves and choose the correct node (i.e., Viral). As seen from Figure 1b, in the Explorer browser this is not possible because the children of Natural are not visible.

Whereas the DOI tree was more forgiving for users' errors, its visual characteristics also had a greater potential for distraction. One example comes from looking at how participants completed the high scent task Find the lobster. Surprisingly, this task took a lot more time in the DOI browser (65s on average) than in the Explorer browser (only 25s). When we examined the data, we noticed that participants in the DOI browser tended to explore the node Fishes and related nodes more than in the Explorer. One explanation is that, because the node Fishes is visible on the screen early in the task (even after clicking on the node Animals) and, due to its high semantic similarity with lobster, it acts as a powerful distractor. Fishes does not have the same visibility in the Explorer, so users are less likely to make the mistake of choosing it. Figure 2 shows an instance where the node Fishes is displayed in a highly visible position.

These data indicated that, as suggested by our initial intuition, a greater amount of information is exposed and processed in the DOI browser. However, this extra information has the potential of distracting the users in their search for a solution to their query. It is interesting that although people could theoretically choose to be efficient in the DOI browser as in the Explorer (in the sense of not wandering too much off the solution path), in some sense they cannot help but absorb all the extra information that is available to them. That process of gathering unneeded information compensates for the potential time gains that the DOI browser 
may bring. In the end, although different mechanisms may characterize browsing in the two types of environment, the DOI browser and the Explorer browser end up being about the same in terms of efficiency.

Direction of eye movements and mouse clicks. Since one of our principal interests was to understand how the visual search interferes with the semantic aspects of the labels in the DOI browser, we also analyzed the eye movement data. We report here only the data from the DOI browser. We split the eye movements and the mouse clicks into two categories: movements/clicks made immediately after the mouse had been clicked (i.e., immediately after the screen has changed and new information has appeared on the screen) and movements/clicks made in between two mouse clicks, when the screen was static. We wanted to understand whether there were any systematic patterns of exploring the screen, so we analyzed the direction of the eye movement/mouse click. We defined three directions: (1) down, if the next action was on a node at a level lower in the tree than the current node; (2) up, if the next action was on a level higher than that of the current one; and (3) lateral if the next action was at the same level.

We found that participants' visual strategy was different in the high and low scent tasks. Immediately after clicking the mouse, participants had a marginal tendency to look up (i.e., back to the root) more in the low scent cases $(t(10)=1.84, p<0.1)$. After a given mouse click, the next mouse click was more likely to be down in the tree for high scent than for low scent tasks $(t(10)=4.93, p<0.001)$ and up or lateral in the tree for low scent tasks (up: $t(10)=$ $-3.77 ; p<0.005$; lateral: $t(10)=-4.37 ; p<0.001)$. These data showed that participants were more likely to follow the path they were on in the high scent cases, whereas in the low scent cases they may have preferred to go back or wander around the tree more in search for a label with a stronger scent.

In between mouse clicks, participants tended to look back up in the tree more for low scent tasks than for high scent tasks $(t(10)=-1.93 ; p<0.1)$. However, for both types of tasks the majority of movements (more than 55\%) were made in the lateral direction (i.e., on the same tree level).

\section{MODELING NAVIGATION}

In this section we present an ACT-R [1] computational model of navigation in degree of interest tree. The ultimate goal of such a model is to capture the data at the very fine grain level of eye movements. While we are not still at the stage where we could report such an accomplishment, we believe there are many lessons to be learned even from trying to model participants' data at a coarser grain level such as response times and nodes clicked or looked at.

Our ACT-R model has two components: a visual search component and a semantic component. The model first decides which part of the screen it needs to focus on (based mostly on visual cues such as screen position, density, etc.) and then it examines the nodes in that part of the screen. The node examination is based on a scent type of function: for each node, it evaluates how good a match it is for this particular target. Then it selects the best node and clicks on it (exceptions may happen, though).

In the next section we discuss how we estimate the scent of a particular node.

\subsection{Estimating Individual Node Scent}

The results of our experiment showed that semantic factors (low versus high scent) are highly relevant for both low and high scent tasks. We saw that people are faster for high than for low scent task. Our assumption is that the high scent targets are on tree paths with high scent nodes, that is, with nodes that look highly relevant to the target (as opposed to other nodes in the tree). But how do we estimate the scent of a node (e.g, Vegetables or People) with respect to a particular target (e.g., Banana)? One way is to ask people directly. This is how it has been done in our experiment in order to decide the scent of different tasks. However, the ratings that were collected referred to only the first four levels in our hierarchy. But when solving the task, since a model (or a person) could get to a random point in the tree, it would be useful to be able to estimate the scent of any node in the tree with respect to the particular task. There are about 7000 nodes in the tree, so it is not feasible to collect human ratings for all those nodes and for all 16 tasks. Therefore, a method for automatically estimating scent would be highly valuable.

Measures of semantic similarity may look as obvious choices for estimations of scent. We first decided to look at LSA [8] and PMI[15] as measures of semantic similarity. Both of these measures essentially compute the similarity between two words based on their co-occurrences in the same documents. They have been used in the past with success (e.g., [2]). Unfortunately, these measures proved to be quite poor choices. Indeed, when we compared them with the human data that we had collected, neither PMI or LSA fared well. for (Out of the 16 targets, only once the humans' top choice matched one of these measures' top choice.). But after looking at the task in more depth, we realized that for our ontology similarity was not a fair measure of scent.

Here is why. Imagine that you had to answer the question: "under which of these labels is it more likely to find the label banana: people, places, things or monkeys"? Probably you would pick up things. However, in sentences we often encounter banana and monkeys or even people together, perhaps because people and monkeys usually eat bananas or because people slip on banana peels. That kind of cooccurrence would be captured by LSA or PMI; one may think of it as measuring semantic associatedness.

So, we thought that instead of picking on associatedness cues, in a search task people may actually pick on category membership. Is banana a thing more than a place, a monkey or a person? Unfortunately, although there are quite a few programs that automatically discover hypernymy, to the best of our knowledge there is none that answers this kind of question.

With that in mind, we decided to build our own program. Much of the work on automatic hypernym discovery $[6,14]$ relies on the observation that there are certain textual patterns that mark the description of a category relationship 
(e.g., banana is a (kind/type of) fruit, fruits such as bananas, fruits especially banana, fruit called banana). We do not have a corpus rich in such examples (and that is one of the problems of automatic hypernym discovery research), so we decided to use the Google's corpus (available via Google API) to collect data about the number of co-occurrences of such strings. For each of our tasks we generated a Google query $\left.{ }^{2}\right)$. This is an example: "arthropods including lobster" OR "arthropods including lobsters" OR "arthropod especially lobster" OR "arthropods especially lobster" OR "arthropods especially lobsters" OR "lobster is a kind of arthropod" OR "lobster is a type of arthropod OR "arthropods like lobster" OR "arthropod called lobster" OR "arthropods called lobsters" OR "lobster and other arthropods" OR "lobsters and other arthropods" OR "lobster or other arthropods" OR "lobsters or other arthropods" OR "arthropod such as lobster OR "arthropods such as lobster" OR "arthropods such as lobsters" OR "lobster is an arthropod" OR "lobster an arthropod". Then we recorded the estimated number of results that Google returned. (Note that this method is applicable with any other big enough corpus and search engine.)

To estimate scent from the numbers returned from Google, for each task we ranked the nodes in the reverse order of the number of results and then assigned a co-occurrence rank to each node. For each node we also computed PMI-like scores by dividing the Google results to the frequency of the node label (this frequency was obtained as the results returned from querying Google with the node label - e.g., "lobster" OR "lobsters") and ranked these numbers in the same way, from highest to lowest. Then we averaged the co-occurrence and the PMI ranks to obtain a scent indicator ${ }^{3}$. This measure resulted in Google's top choice agreeing with humans's top choice in 7 out of 16 tasks. Out of the remaining 9 tasks, six of them presented some overlap between Google's top three choices and humans' top three choices. For only three tasks there was no relationship between this method's top three choices and subjects' top three.

\subsection{Brief Introduction to ACT-R}

ACT-R[1] is a production-system-based cognitive architecture that has been extensively used to model various aspects of human cognition, from memory and problem solving, to language and web browsing. ACT-R resembles a programming language in which all the constructs have some psychological validity. For instance, once such construct is the retrieval of a piece of information from memory. ACT-R imposes various constraints on its constructs; for example, the time needed for a retrieval operation is well specified; also what kinds of items you can retrieve from memory at any given moment. These constraints are backed by many psychology studies and models of human cognition. All the constructs in ACT-R can be put together in a program called a computational model. A computational model for a task is an ACT-R program that does the task and embodies a theory about the mechanisms that humans deploy when solving that task. Because ACT-R specifies a certain granularity

\footnotetext{
${ }^{2}$ In fact, each Google query needed to be split in several queries due to Google-imposed constraints on the length of the query.

${ }^{3}$ The actual scent indicator was the difference between a large constant and that average, since we needed low rank nodes to have high scent values.
}

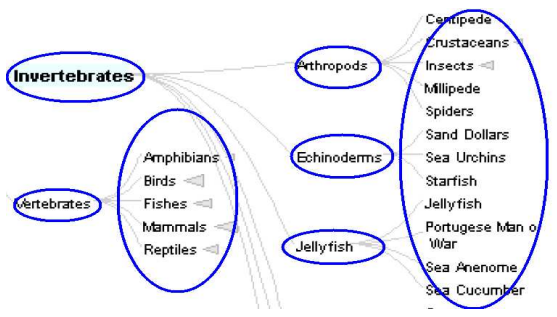

Figure 3: Different proximity-based groups as defined by our model.

(50ms) for the actions (called productions) inside a model and because each other operation takes a certain (not necessarily constant) time specified by the theory (retrieval, moving of visual attention, key press, etc.), we can derive quantitative predictions (i.e., response time, accuracy) from the model and we can compare those predictions with human data.

ACT-R operates with two kinds of knowledge: declarative and procedural. Procedural knowledge refers to actions that we normally take to accomplish a task (e.g., turn on the key in the ignition to start the car). Declarative knowledge refers to facts that we know (e.g., that "Paris is the capital of France" or that "banana is a member of the category fruits"). These facts are atomic "pieces" of memory called chunks. Each chunk is characterized by an activation level that reflects how often and how recently the chunk has been used. Basic facts such as " $1+2=3$ " typically have very high activation, whereas more obscure facts (e.g., "Stravinsky composed The Nightingale") may have low activation levels. Activation characterizes how available that chunk is: high activation chunks can be retrieved quickly, whereas low activation chunks are harder to retrieve. When ACT$R$ needs to retrieve information from memory, it picks up the chunk that has the highest activation and that satisfies the retrieval constraint. (For instance, assume that ACT$R$ needs to retrieve the capital of China; there maybe two chunks - one expressing the fact that "Beijing is the capital of China" and another that "Peking is the capital of China"; which one is retrieved depends on their relative activations, which possibly differ from individual to individual.) Sometimes the activation of a chunk may be too low (under a certain value called retrieval threshold) to be retrieved.

\subsection{The Model}

According to work in the visual search literature [9, 5], when processing a visual scene, people often tend to group visual items together, select one such group and attend the items within it. The way these visual groups are formed may be based on proximity or based on other common feature that they share to a large degree (e.g., all gray items on a page may form a group and all red items may form a different group). In our model, we assume that the visual display is partitioned according to (vertical) closeness. Figure 3 shows an example of how the visual items are grouped together. Given a display, the model selects the best group according to several heuristics. Groups that contain nodes that are children of the node clicked on are preferred; also sparse groups [5] and groups close to the current eye position (i.e., the node that has been attended last). There is a certain 
inhibition to return back to groups that were visited before. ACT-R does not have such a grouping mechanism available; we have explicitly implemented it. Once a group is selected, the nodes within that group are attended one by one, from top position to bottom position ${ }^{4}$. In agreement to the ACT$\mathrm{R}$ theory, the processing of each node includes three stages: (1) finding the location of the next item; (2) attending that location; (3) encoding the semantic information existent at that location. This last step is equivalent with processing the text of the label; it involves assessing whether the label stands for a hypernym of the search target. The information whether a word is a hypernym of another is encoded in memory in the form of a chunk. The activation of that chunk is proportional with the degree in which the search target is a hyponym of the node (i.e., with the scent of the node, as estimated through the Google search method discussed previously). If the node is a hypernym in a high enough degree, that node will have an activation over the retrieval threshold and will be retrieved. As the model scans the nodes, if it does not encounter any hypernyms and if it's deep enough in the tree ${ }^{5}$, it decreases the probability of continuing to attend nodes in the same visual group. When all the nodes in the group have been visited or when the probability of continuing becomes too low, the model makes a selection: it chooses the node among the previously visited nodes that is the best hypernym of the target ${ }^{6}$. If the model can find a best hypernym in the current visual group, it is the node that will be next clicked on. Otherwise, if no such node exists or if the best hypernym is part of a different visual group ${ }^{7}$ the model makes a probabilistic decision: either leaves the group without clicking and looks for another group to attend or returns to the best hypernym found so far (if it is still displayed on the screen). The model then goes back to selecting a visual group.

Occasionally, when expanding leaves in the hierarchy tree, none of the nodes in the visual group is a hypernym (but they can be members of the same category as the target). For instance, when searching for bananas, one would come across a visual group containing avocado, breadfruit, guava, kiwi, papaya. For leaves (which are marked visually differently than the interior nodes in the DOI visualization), instead of category information, regular semantic similarity (PMI scores) is used. The intuition is that if you are in a part of the tree with leaves similar to what you are looking for (e.g., papaya, kiwi when searching for banana), you are more likely to encounter it than if you are around leaves

\footnotetext{
${ }^{4}$ Although we do not discuss this analysis in our experiment section, this strategy is consistent with our eye movement data. When doing a quadrant analysis of eye movements, after a mouse click, the eyes tend to move first in the northeast direction.

${ }^{5}$ The nodes that are close to the root are very general and unrelated (e.g., level one contains nodes such as People, Places, Events, Things. A node not being part of one of these categories does not predict well whether it is going to be part of another.

${ }^{6}$ The model attempts to retrieve a hypernym; due to the ACT-R's retrieval mechanism, the hypernym with the highest activation (i.e., highest scent with respect to that target) is automatically retrieved.

${ }^{7}$ This is equivalent with the model remembering that elsewhere it has met a better hypernym than any node in this group.
}

that are completely unrelated (e.g., Aquarius, Aries, Cancer, Capricornus). Thus the model processes leaves slightly differently than regular interior nodes. Instead of trying to retrieve whether the leaf is a hypernym of a target or not, it just assesses how similar the link is to the target. In a given group, each time it meets a leaf with low similarity, it decreases the probability to continue. If the probability is too low, the model makes a selection (in case any hypernyms were also included in that group) and/or switches to a different visual group, as before.

Results of the simulations. As discussed in the beginning of this section, we plan to refine this model to capture subjects' data at the level of eye movement sequences. Here we present some of the results obtained so far regarding response times and mouse clicks per task and we compare these data with the human participants data.

Given that the model attempts to predict solution for complex search problems and no correction to the model are made when it makes a "wrong" move (i.e., a move that a human did not do), even finding the solution to a problem is a big accomplishment, especially in a world where the scent of the nodes (as we computed them) can be only approximations of the knowledge that people actually have. We stopped the model after it ran 300s without finding a solution. In this circumstances, the model found solutions to 11 out of the 16 tasks ( 7 high scent, 4 low scent). Part of the problem was our estimation of hypernymy: for instance, the phrase "crosscut saw is a type of $\langle A\rangle$ " is quite rare even for the huge Google corpus. In such cases we tried to estimate the hypernymy of the parts, but again "saw", with its more frequent verb meaning, drove us into problems.

When we looked at the response times, the model was able to capture the difference between low and high scent task: the high scent tasks took on average $41.17 \mathrm{~s}$ and the low scent tasks were slower: 74.88 s for the model. (Human data can be found in Table 1.) On average the model clicked on $69 \%$ of the nodes that humans clicked on $^{8}$. In the case of high scent tasks, the model clicked on $68 \%$ of the nodes that the participants clicked on; whereas this number was $73 \%$ for the low scent tasks.

\section{CONCLUSIONS}

We have presented an experiment and a computational model intended to understand how people navigate through large hierarchical information structures such as DOI trees. The experiment studied how people complete search tasks in two browsers: a DOI tree browser and a Windows-Explorer like browser. The results suggested that, in terms of task completion speed, the two browsers are not different. What really made a difference in the task completion speed was the semantic aspect captured by the information scent: how well chosen were the labels for the nodes. People tend to visit more new nodes in the DOI tree than in the Explorer browser, whereas in the Explorer browser they tend to revisit the same nodes more. By displaying more information,

${ }^{8}$ But some of the clicks that only the model made are perfectly plausible: for instance, when searching for the Pawnee Indian tribe it clicked on Native American, although it so happened that none of the six participants who completed that task clicked on that node. 
the DOI browser seems to encourage users to wander more off the solution path and to gather more knowledge than necessary for task completion. In other words, what time is lost with revisitation (due to backtracking) in the Explorer browser it is spent with exploration of new, unnecessary nodes in the DOI browser. Thus, the DOI browser may prove beneficial over long term usage because it allows users to gather more information about the hierarchy structure. The DOI browser also allows users to recover more easily when they made certain types of errors; however, it also can distract the participants with highly salient but irrelevant items that are placed close to the users' focus of attention.

We also sketched an ACT-R computational model of navigation in DOI trees. The model combined a detailed visual strategy in which a region of the screen was located as relevant with a semantic process that made the selection of which node to click next. The semantic part of the model was based on an automatic estimation of the information scent of the nodes in the tree. In fact, one of the lessons from this model is that, if you want to capture the human performance even in the roughest detail (e.g., which tasks get completed in a useful time) you need a good measure of information scent. We settled on the a measure that captures the hypernymy relationship: in what degree the search target $\mathrm{A}$ is member of the category denoted by the node label $\mathrm{B}$ ? That measure was computed by querying a large corpus (in our case Google's database) with queries describing category membership. Whereas the model was able to capture some of the aspects in the data (e.g., difference in response times for low and high scent task), it is still work in progress. We hope to look at finer grain measures such as individual user performance and sequences of eye movements.

The ultimate goal of this paper was to understand the interplay between visual search and semantic information. We noted earlier that information scent really plays the most important part in search. However (and our earlier stages of the model stood proof for that), even good information scent will not be able to salvage a poor search strategy. Simple facts (such as the fact that people know that if they clicked a node, they should attend to new parts of the screen first) are not trivial to capture in a visual attention model when the visual environment is so rich in items. Subtle semantic cues interfere with the visual attention process (e.g., before, I clicked on a node that was uninteresting so the parts around it on the screen will probably be uninteresting in the future too; or, this node has high scent, therefore I should attend to the visual group that contains its children first) and are hard to capture in a model that has constraints on the working memory capacity (how much information can you remember about the many nodes on the screen?).

Stepping back into the world of focus + context visualizations, our results with the DOI tree browser corroborated those in [13]: cramming more information on the screen does not necessarily improve performance. Putting the right labels on the information that you display is a better way to speed up the task.

\section{ACKNOWLEDGMENTS}

Portions of this research have been funded by an Advanced Research and Development Activity, Novel Intelligence from
Massive Data Program, Contract No. MDA904-03-C-0404 to S.K. Card and P. Pirolli. We thank Duncan Brumby and Jeff Heer for their substantial help with integrating the DOI browser with the ACT-R architecture.

\section{ADDITIONAL AUTHORS}

Additional authors: Julie Heiser (Stanford University, Stanford CA 94305, email: jheiser@psych.stanford.edu).

\section{REFERENCES}

[1] J. Anderson and C. Lebiere. The atomic components of thought. Erlbaum, Mahwah, NJ, 1998.

[2] R. Budiu and J. Anderson. Interpretation-based processing: A unified theory of semantic sentence comprehension. Cognitive Science, 28:1-44, 2004.

[3] S. Card, J. Mackinlay, and B. Schneiderman. Information visualization. Morgan Kaufmann, 1999.

[4] S. Card and D. Nation. Degree-of-interest trees: a component of an attention-reactive user interface. In Advanced Visual Interface '02, Trento, Italy, 2002.

[5] T. Halverson and A. J. Hornof. Strategy shifts in mixed-density search. In The 26th Annual Meeting of the Cognitive Science Society, Chicago, IL, 2004.

[6] M. Hearst. Automatic acquisition of hyponyms from large text corpora. In Fourteenth International Conference on Computational Linguistics, 1992.

[7] J. Lamping, R. Rao, and P. Pirolli. A focus + context technique based on hyperbolic geometry for visualizing large hierarchies. In Conference on Human Factors in Computing Systems CHI '95, 1995.

[8] T. K. Landauer and S. T. Dumais. A solution to Plato's problem: the Latent Semantic Analysis theory of acquisition, induction and representation of knowledge. Psychological Review, 104:211-240, 1997.

[9] G. Logan. The code theory of visual attention: An integration of space-based and object-based attention. Psychological Review, 103:603-649, 1996.

[10] R. Lorch and J. Myers. Regression analyses of repeated measures data in cognitive research. Journal of Experimental Psychology: Learning, memory and cognition, 16(1):149-157, 1990.

[11] K. Mullet, C. Fry, and D. Schiano. On your marks, get set, browse! In Human Factors in Computer Systems, CHI '97 (Extended abstracts), Atlanta, 1997.

[12] P. Pirolli and S. Card. Information foraging. Psychological Review, 1999.

[13] P. Pirolli, S. Card, and M. Van Der Wege. The effect of information scent on searching information visualizations of large tree structures. In $A V I$, Palermo, Italy, 2000.

[14] R. Snow, D. Jurafsky, and A. Y. Ng. Learning syntactic patterns for automatic hypernym discovery. In NIPS 17, 2005.

[15] P. Turney. Mining the web for synonyms: PMI-IR versus LSA on TOEFL. In Twelfth European Conference on Machine Learning (ECML-2001), 2001. 